\title{
CONTROLS INSENSITIZING THE OBSERVATION OF A QUASI-GEOSTROPHIC OCEAN MODEL*
}

\author{
ENRIQUE FERNÁNDEZ-CARA ${ }^{\dagger}$, GALINA C. GARCIA ${ }^{\ddagger}$, AND AXEL OSSES ${ }^{\S}$
}

\begin{abstract}
We consider a linear quasi-geostrophic ocean model with partially known initial conditions. We search for controls that make the observation locally insensitive to the perturbations of the initial data. Their existence is equivalent to the null controllability property for an associated cascade Stokes-like system. Thanks to the presence of the Coriolis term, we are able to prove the existence of such controls. Our strategy is the following. First, we prove a unique continuation property for the adjoint of the state system that leads to approximate controllability; then, under certain assumptions, an observability inequality is established for the adjoint. The proof is inspired by the arguments leading to the unique continuation property. This inequality leads to the desired null controllability result.
\end{abstract}

Key words. insensitizing controls, Carleman inequalities, unique continuation, null controllability, ocean model

AMS subject classifications. 93B05, 35B37, 35B60, 35Q30

DOI. $10.1137 / \mathrm{S} 0363012903433607$

\section{Introduction and main results.}

1.1. Incomplete initial data ocean model. Let $\Omega$ be a nonempty open bounded and connected subset of $\mathbb{R}^{2}$, with boundary $\Gamma$ of class $\mathcal{C}^{2}$ and outwards unit normal vector $\nu=\nu(x)$. Let $\omega$ be a nonempty open subset of $\Omega, T>0, Q=\Omega \times(0, T)$, and $\Sigma=\Gamma \times(0, T)$. In this paper, we will consider a linear quasi-geostrophic ocean model $[1,15,16]$ described by the following equations:

$$
\left\{\begin{array}{l}
u_{t}-A \Delta u+\gamma u+\left(f_{0}+\beta x_{2}\right) \mathrm{k} \wedge u+\frac{1}{\rho_{0}} \nabla p=\mathcal{T}+h 1_{\omega} \quad \text { in } Q, \\
\operatorname{div} u=0 \text { in } Q \\
u=0 \text { on } \Sigma, \\
u(0)=u_{0}+\tau \widehat{u}_{0} \quad \text { in } \Omega,
\end{array}\right.
$$

where $u(x, t)$ and $p(x, t)$, respectively, denote the velocity and the pressure of the fluid at $(x, t)=\left(x_{1}, x_{2}, t\right) \in \mathbb{R}^{2} \times \mathbb{R}_{+}$. In this model, $A$ represents the horizontal eddy viscosity coefficient, $\gamma$ is the bottom friction coefficient, $\rho_{0}$ is the fluid density, and $\left(f_{0}+\beta x_{2}\right) \mathrm{k} \wedge u$ is the Coriolis term, with $\mathrm{k} \wedge u=\left(-u_{2}, u_{1}\right)$. In the right-hand side, $1_{\omega}$ denotes the characteristic function of $\omega$ and $\mathcal{T}$ is a given source in $L^{2}(Q)^{2}$. The term

${ }^{*}$ Received by the editors August 22, 2003; accepted for publication (in revised form) July 1, 2004; published electronically March 11, 2005.

http://www.siam.org/journals/sicon/43-5/43360.html

${ }^{\dagger}$ Departamento de Ecuaciones Diferenciales y Análisis Numérico, Universidad de Sevilla, Aptdo. 1160, 41080 Sevilla, Spain (cara@numer.us.es). This author's work was partially supported by D.G.E.S. (Spain) grants BFM2000-1317 and BFM2003-06446.

¥Facultad de Ingeniería, Universidad Católica de la Santísima Concepción, Casilla 297, Concepción, Chile (galina@ucsc.cl). This author's work was supported by FONDAP in Applied Mathematics, CONICYT Ph.D. grants, and CONICYT-INRIA cooperation agreements (Chile).

${ }^{\S}$ Departamento de Ingeniería Matemática, Universidad de Chile, Casilla 170/3 Correo 3, Santiago, Chile, and Centro de Modelamiento Matemático, UMI 2807/Universidad de Chile-CNRS, Santiago, Chile (axosses@dim.uchile.cl). This author's work was partially supported by FONDAP in Applied Mathematics, FONDECYT-CONICYT 1030808-7030059, and ECOS-CONICYT C01E02 grants (Chile). 
$\tau \widehat{u}_{0}$, where $\tau \in \mathbb{R}$, represents a small unknown perturbation of the initial velocity field $u_{0}$, and $h=h(x, t)$ is a control function to be determined.

Notice that the Coriolis force is represented by a zero order coupling term in the equations. It introduces a different behavior of the system depending on the direction in space. To simplify the presentation of the results, we will assume that $A=1, \gamma=1$, $f_{0}=1, \beta=1$, and $\rho_{0}=1$.

We introduce the following spaces, which are usual in the analysis of Stokes systems:

$$
\begin{aligned}
& H=\left\{v \in L^{2}(\Omega)^{2}: \quad \operatorname{div} v=0 \text { in } \Omega, v \cdot \nu=0 \text { on } \Gamma\right\}, \\
& V=\left\{v \in H_{0}^{1}(\Omega)^{2}: \quad \operatorname{div} v=0 \text { in } \Omega\right\}, \quad W=H^{2}(\Omega)^{2} \cap V .
\end{aligned}
$$

Recall that

$$
W \hookrightarrow V \hookrightarrow H \equiv H^{\prime} \hookrightarrow V^{\prime} \hookrightarrow W^{\prime},
$$

where the embeddings are dense and compact.

For any given $u_{0}, \tau \widehat{u}_{0} \in H$ with $\left\|\widehat{u}_{0}\right\|_{0, \Omega}=1$, any $\mathcal{T} \in L^{2}(Q)^{2}$, and any $h \in$ $L^{2}(\omega \times(0, T))^{2}$, the linear system (1.1) possesses a unique solution $(u, p)$, with $u \in$ $L^{2}(0, T ; V) \cap H^{1}\left(0, T ; V^{\prime}\right)$ and $p \in W^{-1, \infty}\left(0, T ; L^{2}(\Omega)\right)$. ( $p$ is unique up to an additive distribution only depending on $t$.) This is easily proved by adapting the arguments of [17] to the presence of a skew-symmetric Coriolis term in the equations. Notice that if we had $u_{0}+\tau \widehat{u}_{0} \in V$, then the couple $(u, p)$ would satisfy $u \in L^{2}(0, T ; W) \cap H^{1}(0, T ; H)$ and $p \in L^{2}\left(0, T ; H^{1}(\Omega)\right)$.

We will be concerned with the search of controls such that the velocity measurements over an observation set are either insensitive or almost insensitive to small variations of the initial conditions. To do this, we will use insensitizing control theory.

1.2. Insensitizing controls and controllability. Let $\mathcal{O}$ be an open nonempty subset of $\Omega$ and let us introduce the following functional, defined on the family of solutions to (1.1):

$$
\Phi(u)=\frac{1}{2} \int_{0}^{T} \int_{\mathcal{O}}|u(x, t)|^{2} d x d t .
$$

The notion of insensitizing controls was introduced by Lions [13]. In the context of (1.1)-(1.2), it reads as follows. if

Definition 1.1. We say that the control $h \in L^{2}(\omega \times(0, T))^{2}$ is $\Phi$ insensitizing

$$
\left.\frac{d}{d \tau} \Phi(u)\right|_{\tau=0}=0 \quad \forall \widehat{u}_{0} \in H \quad \text { with }\left\|\widehat{u}_{0}\right\|_{0, \Omega}=1 .
$$

On the other hand, we say that $h \in L^{2}(\omega \times(0, T))^{2}$ is $\Phi \varepsilon$-insensitizing if

$$
\left|\frac{d}{d \tau} \Phi(u)\right|_{\tau=0} \mid \leq \varepsilon \quad \forall \widehat{u}_{0} \in H \quad \text { with }\left\|\widehat{u}_{0}\right\|_{0, \Omega}=1 .
$$

Of course, in (1.3) and in (1.4) $u$ is, together with $p$, the solution to (1.1).

The $\Phi$ insensitizing (resp., $\Phi \varepsilon$-insensitizing) controls $h$ must be interpreted as those leading to an observation $\Phi(u)$ that is locally independent (resp., almost independent) at the initial perturbation $\tau \widehat{u}_{0}$. The existence of such controls is a pertinent 
question, since it is realistic to assume that the true initial conditions for (1.1) are unknown. In fact, as noticed in [13], it would be more convenient to search for $\Psi$ insensitizing (or $\Psi \varepsilon$-insensitizing) controls, where

$$
\Psi(u)=\frac{1}{2} \int_{0}^{T} \int_{\mathcal{O}}|\operatorname{curl} u(x, t)|^{2} d x d t
$$

but this is beyond the scope of this article and will be the subject of future work.

It is easy to characterize the insensitivity (resp., $\varepsilon$-insensitivity) property in terms of exact null controllability (resp., approximate controllability) of a related cascade system. Indeed, let $(\bar{u}, \bar{p})$ and $(q, r)$ be the solutions of the following systems:

$$
\begin{aligned}
& \left\{\begin{array}{l}
\bar{u}_{t}-\Delta \bar{u}+\bar{u}+\left(1+x_{2}\right) k \wedge \bar{u}+\nabla \bar{p}=\mathcal{T}+h 1_{\omega} \text { in } Q, \\
\operatorname{div} \bar{u}=0 \text { in } Q, \\
\bar{u}=0 \text { on } \Sigma, \\
\bar{u}(0)=u_{0} \text { in } \Omega,
\end{array}\right. \\
& \left\{\begin{array}{l}
-q_{t}-\Delta q+q-\left(1+x_{2}\right) k \wedge q+\nabla \pi=\bar{u} 1_{\mathcal{O}} \text { in } Q, \\
\operatorname{div} q=0 \text { in } Q, \\
q=0 \text { on } \Sigma, \\
q(T)=0 \text { in } \Omega .
\end{array}\right.
\end{aligned}
$$

Then the control $h$ is $\Phi$ insensitizing (resp., $\Phi \varepsilon$-insensitizing ) if and only if

$$
q(0)=0 \quad\left(\text { resp., }\|q(0)\|_{0, \Omega} \leq \varepsilon\right)
$$

Indeed, in view of (1.2), condition (1.3) is equivalent to

$$
\left.\int_{0}^{T} \int_{\mathcal{O}} \bar{u} \cdot u_{\tau} d x d t=0 \quad \text { (resp., (1.4) is equivalent to }\left|\int_{0}^{T} \int_{\mathcal{O}} \bar{u} \cdot u_{\tau} d x d t\right| \leq \varepsilon\right),
$$

where $\bar{u}$ is the solution of (1.5) and $u_{\tau}$ is the solution of (1.1) differentiated with respect to $\tau$. Using the definition of $(q, \pi)$ and integrating by parts, we obtain

$$
\int_{\Omega} q(0) \cdot \widehat{u}_{0} d x=0 \quad\left(\text { resp. },\left|\int_{\Omega} q(0) \cdot \widehat{u}_{0} d x\right| \leq \varepsilon\right) \quad \forall \widehat{u}_{0} \in H \quad \text { with }\left\|\widehat{u}_{0}\right\|_{0, \Omega}=1 .
$$

This is equivalent to (1.7). See [18] for more detail.

Notice that since $\bar{u} \in L^{2}(0, T ; V)$, we also have $q \in L^{2}(0, T ; W) \cap H^{1}(0, T ; H)$ and $\pi \in L^{2}\left(0, T ; H^{1}(\Omega)\right)$.

We are thus in the presence of a null controllability problem (resp., an approximate controllability problem) for a cascade system, where the control $h$ is not acting directly in the system satisfied by $q$ (the function we want to drive to zero after a time interval of length $T$ ) but indirectly, through $\bar{u} 1_{\mathcal{O}}$.

1.3. Main results. There have been several recent results concerning the existence of insensitizing and $\varepsilon$-insensitizing controls for parabolic problems.

Thus, in [2] the existence of $\varepsilon$-insensitizing controls for linear heat equations with partially known initial and boundary conditions was established. The same was also obtained for semilinear heat equations with globally Lipschitz-continuous nonlinearities. Since then, it has been proved in [18] that insensitizing controls exist for the same equations completed with zero initial data, under suitable assumptions 
on the source term. In [3], the authors extended these results to other more general (slightly superlinear) nonlinearities.

In this paper, we deal with the insensitizing and $\varepsilon$-insensitizing problems for the case of the Stokes-type equations (1.1). Our results were sketched in [7]. These are the first insensitivity results in the literature for equations of this type, as far as we know.

We will assume that the following geometrical hypothesis is satisfied, as in the previous references:

$$
\omega \cap \mathcal{O} \neq \emptyset .
$$

Our main results are the following.

THEOREM 1.2. Let $T>0$ and assume that (1.8) is satisfied. Then, for each $\varepsilon>0$ there exists a control $h \in L^{2}(\omega \times(0, T))^{2}$ which is $\Phi$-insensitizing.

THEOREM 1.3. Under the assumptions of Theorem 1.2, if we also have $u_{0}=0$ and

$$
\int_{0}^{T} \int_{\Omega} \exp \left(M t^{-4}\right) \mathcal{T}^{2} d x d t<+\infty
$$

for an appropriate constant $M$ depending on $\Omega, \omega, \mathcal{O}$, and $T$, then there exists a control $h \in L^{2}(\omega \times(0, T))^{2}$ which is $\Phi$ insensitizing.

It was proved in [18] for the linear heat equation that, in general, we cannot expect the existence of insensitizing controls for nonvanishing initial data in $L^{2}(\Omega)$ when $\Omega \backslash \bar{\omega} \neq \emptyset$. The proof of this result is based on a counterexample for which the appropriate observability inequality fails when the initial data belong to $L^{2}(\Omega)$. Similar arguments could be used for Stokes systems. In view of this, it is reasonable to impose in Theorem 1.3 that $u_{0}=0$.

This paper is organized as follows. In section 2, we prove Theorem 1.2, where we obtain a unique continuation result for an adjoint cascade system thanks to the presence of the Coriolis term. In section 3, we prove Theorem 1.3. In this section, we show that insensitizing controls do exist if an appropriate observability inequality holds. We deduce this observability inequality in section 3.2 by means of an appropriate global Carleman inequality for the same adjoint cascade system. The proof of this global Carleman inequality is given in section 3.1 and follows a chain of estimates based on the steps of the unique continuation proof. At the end of this section and to be self-contained, we give the proof of a standard global Carleman estimate for Stokes-like systems that is needed in section 3.1. Finally, in section 4, we summarize the key points of this article in some final remarks.

2. Proof of Theorem 1.2. We can assume without loss of generality that $\mathcal{T}=0$ and $u_{0}=0$ in (1.5)-(1.6). It is well known that the existence of $\varepsilon$-insensitizing controls for (1.5)-(1.6) is equivalent to a unique continuation property of the associate adjoint system

$$
\begin{aligned}
& \left\{\begin{array}{l}
\phi_{t}-\Delta \phi+\phi+\left(1+x_{2}\right) k \wedge \phi+\nabla \theta=0 \text { in } Q, \\
\operatorname{div} \phi=0 \text { in } Q, \\
\phi=0 \text { on } \Sigma, \\
\phi(0)=\phi_{0} \text { in } \Omega,
\end{array}\right. \\
& \left\{\begin{array}{l}
-z_{t}-\Delta z+z-\left(1+x_{2}\right) k \wedge z+\nabla r=\phi 1_{\mathcal{O}} \text { in } Q, \\
\operatorname{div} z=0 \text { in } Q, \\
z=0 \text { on } \Sigma, \\
z(T)=0 \text { in } \Omega
\end{array}\right.
\end{aligned}
$$


for a given $\phi_{0} \in H$. This coupled system possesses a unique solution $(\phi, \theta),(z, r)$, with at least $\phi, z \in L^{2}(0, T ; V) \cap H^{1}\left(0, T ; V^{\prime}\right)$ and $\theta, r \in W^{-1, \infty}\left(0, T ; L^{2}(\Omega)\right)$. (Again, $\theta$ and $r$ are unique up to a distribution depending only on $t$.)

Using (1.5)-(1.6) and (2.1)-(2.2) the following duality identity is easily deduced:

$$
\int_{0}^{T} \int_{\omega} h \cdot z d x d t=\int_{\Omega} q(0) \cdot \phi_{0} d x \quad \forall h \in L^{2}(\omega \times(0, T))^{2} .
$$

It is clear from this last identity that the set $\left\{q(0): h \in L^{2}(\omega \times(0, T))^{2}\right\}$ is dense in $H$ if the following unique continuation result holds.

Lemma 2.1. Assume (1.8). Let $(\phi, \theta),(z, r)$ be the solution to (2.1)-(2.2) with $\phi_{0} \in H$. Then, if $z=0$ in $\omega \times(0, T)$, we necessarily have $z \equiv \phi \equiv 0$ and $\nabla r \equiv \nabla \theta \equiv 0$ in $Q$.

Proof. This is a direct consequence of a more general unique continuation result. To state this result precisely, let $\widetilde{\omega}=\omega \cap \mathcal{O} \neq \emptyset$ and let us set

$$
C_{1}(\widetilde{\omega})=\left\{\left(x_{1}, x_{2}\right) \in \Omega: \exists x_{1}^{0} \text { s.t. }\left(x_{1}^{0}, x_{2}\right) \in \widetilde{\omega}\right\}, \quad \Sigma_{1}(\widetilde{\omega})=\left(\Gamma \cap \bar{C}_{1}\right) \times(0, T) .
$$

$\left(C_{1}(\widetilde{\omega})\right.$ is the horizontal component of $\widetilde{\omega}$.) We will prove that if $\phi=\left(\phi_{1}, \phi_{2}\right)$ is together with $\theta, z$, and $r$ a solution of

$$
\begin{aligned}
& \left\{\begin{array}{l}
\phi_{t}-\Delta \phi+\phi+\left(1+x_{2}\right) k \wedge \phi+\nabla \theta=0 \text { in } Q, \\
\operatorname{div} \phi=0 \text { in } Q, \\
\phi_{1}=0 \text { on } \Sigma_{1}
\end{array}\right. \\
& \left\{\begin{array}{l}
-z_{t}-\Delta z+z-\left(1+x_{2}\right) k \wedge z+\nabla r=\phi 1_{\widetilde{\omega}} \quad \text { in } Q, \\
\operatorname{div} z=0 \text { in } Q,
\end{array}\right.
\end{aligned}
$$

and $z=0$ in $\widetilde{\omega} \times(0, T)$, then $\phi \equiv 0$.

To prove this assertion, we divide the proof into two steps. Without loss of generality, we can assume that $\widetilde{\omega}$ is connected; otherwise we would replace $\widetilde{\omega}$ by one of its connected components.

In a first step, we deduce from the fact that $z=0$ in $\widetilde{\omega} \times(0, T)$ that $\phi_{2}=0$ and $\phi_{1}$ is constant if they are restricted to $\widetilde{\omega} \times(0, T)$. Thus, since $z=0$ in $\widetilde{\omega} \times(0, T)$ we notice that $\operatorname{curl} \phi=0$ in $\widetilde{\omega} \times(0, T)$ by applying the curl operator in the equation of $z$ in (2.4). Using this fact, if we now apply the curl operator to the first equation in (2.4), thanks to the presence of the Coriolis term we obtain that

$$
\operatorname{curl}\left(\left(1+x_{2}\right) k \wedge \phi\right)=\phi_{2}+\operatorname{div} \phi=\phi_{2}=0
$$

in $\widetilde{\omega} \times(0, T)$. Now, since $\operatorname{div} \phi=0$ and $\operatorname{curl} \phi=0$ in $\widetilde{\omega} \times(0, T)$, we have $\nabla \phi_{1}=0$ in $\widetilde{\omega} \times(0, T)$. Therefore $\phi_{1}$ is constant in $\widetilde{\omega} \times(0, T)$ and we certainly obtain $\phi=($ Const., 0$)$ in $\widetilde{\omega} \times(0, T)$.

In a second step, let us introduce

$$
\psi=\frac{\partial \phi}{\partial x_{1}}, \quad \pi=\frac{\partial \theta}{\partial x_{1}}
$$

and the coefficient matrix:

$$
a=\left[\begin{array}{cc}
1 & -\left(1+x_{2}\right) \\
\left(1+x_{2}\right) & 1
\end{array}\right] \in L_{l o c}^{\infty}(Q) .
$$


Then we have

$$
\begin{aligned}
& \left\{\begin{array}{l}
\psi_{t}-\Delta \psi+a \psi+\nabla \pi=0 \quad \text { in } Q, \\
\operatorname{div} \psi=0 \text { in } Q,
\end{array}\right. \\
& (\psi, \pi) \in L_{l o c}^{2}(Q)^{2} \times \mathcal{D}^{\prime}(Q)
\end{aligned}
$$

with $\psi=0$ in $\widetilde{\omega} \times(0, T)$. Here, we use a sharp uniqueness property for the Stokes system (2.7) proved in [5] that says that, under the regularity determined by (2.6) and (2.8), one has $\psi \equiv 0$ in $Q$. Now, from (2.5) we obtain $\partial \phi_{i} / \partial x_{1} \equiv 0$ for $i=1,2$ in $Q$. Since $\operatorname{div} \phi=0$ in $Q$ we also have $\nabla \phi_{2}=0$ in $Q$ and, from the fact that $\phi_{2}=0$ in $\widetilde{\omega} \times(0, T)$, we deduce that $\phi_{2} \equiv 0$ in $Q$ (recall that $\Omega$ is connected).

On the other hand, since $\partial \phi_{1} / \partial x_{1}=0$ in $Q$ and $\phi_{1}=0$ on $\Sigma_{1}$, we see that $\phi_{1}=0$ in $C_{1} \times(0, T)$. Finally we have $\phi=\left(\phi_{1}, \phi_{2}\right)=0$ in $C_{1} \times(0, T)$, which is an open subset of $Q$. We can conclude that $\phi \equiv 0$ in $Q$ using again the uniqueness property of [5]. (We can also use here the weaker result proved in [4].)

Remark 1. The method used in the second part of the proof of Lemma 2.1 leads to the following uniqueness property in any dimension $n$. Let $(\phi, \theta)$ be the solution of

$$
\left\{\begin{array}{l}
\phi_{t}-\Delta \phi+a \phi+\nabla \theta=0 \quad \text { in } Q, \\
\operatorname{div} \phi=0 \text { in } Q, \\
\phi=0 \text { on } \Sigma_{1}(\omega),
\end{array}\right.
$$

where $Q=\Omega \times(0, T), \Omega$ is a nonempty open bounded connected subset of $\mathbb{R}^{n}, \omega$ is an open nonempty subset of $\Omega, \Sigma_{1}$ and $C_{1}$ are as defined in (2.3), and $a \in L^{\infty}(Q)$. If $a$ is a function independent of $x_{1}$ in $Q$ and $\phi$ is independent of $x_{1}$ in $\omega \times(0, T)$, then $\phi$ vanishes in $Q$. Indeed, let us introduce $\psi=\partial \phi / \partial x_{1}, \pi=\partial \theta / \partial x_{1}$, which satisfy a Stokes problem similar to (2.9), and this problem does not involve $\phi$ explicitly since $a$ is independent of $x_{1}$. Now, from the uniqueness property in [5], $\psi \equiv 0$ in $Q$. Consequently $\partial \phi / \partial x_{1}=0$ in $Q$ and $\phi=0$ on $\Sigma_{1}$, so we have $\phi=0$ in $C_{1} \times(0, T)$. Using the unique continuation property in [5] once again, we obtain that $\phi \equiv 0$ in $Q$.

Remark 2. The previous remark shows that the Coriolis term plays a crucial role only in the first part of the proof of Lemma 2.1. In fact, the presence of the Coriolis term allows us to prove that $\operatorname{curl} \phi=0$ in $\widetilde{\omega} \times(0, T)$ implies that the second component of $\phi$ vanishes in $\widetilde{\omega} \times(0, T)$. This will also be important in the deduction of the Carleman inequality later.

Remark 3. In the proof of the previous lemma, it is not possible to use the results of [4] concerning uniqueness properties of the Stokes system when one of the components of $\phi$ vanishes in $\widetilde{\omega} \times(0, T)$. This is because the results in [4] require that the coefficient $a$, introduced in (2.6), satisfy $a_{12}=0$.

3. Proof of Theorem 1.3. The proof of the existence of insensitizing controls for (1.1), i.e., the exact null controllability for (1.5)-(1.6), relies on the following observability result for the cascade adjoint system $(2.1)-(2.2)$.

Proposition 3.1. Assume that $\omega \cap \mathcal{O} \neq \emptyset$. There exist positive constants $M$ and $K$, depending only on $\Omega, \omega, \mathcal{O}$, and $T$, such that the inequality

$$
\int_{0}^{T} \int_{\Omega} \exp \left(-M t^{-4}\right)|z|^{2} d x d t \leq K \int_{0}^{T} \int_{\omega}|z|^{2} d x d t
$$

holds for every solution of (2.1)-(2.2) with $\phi_{0} \in H$.

The proof of this result is based on a global Carleman inequality (see Theorem 3.3), as will be seen in section 3.2. This Carleman inequality will be proved in section 3.1. 
Let us now give the proof of Theorem 1.3 using Proposition 3.1. Thus, let us assume that (1.8) is satisfied, $u_{0}=0$, and (1.9) holds with $M$ being the constant furnished by Proposition 3.1.

The approximate control $h$ of minimal norm in $L^{2}(\omega \times(0, T))^{2}$ corresponding to $u_{0}=0$, a source term $\mathcal{T}$ satisfying (1.9), and tolerance $\varepsilon>0$ can be obtained by minimizing in $L^{2}(\Omega)^{2}$ the following convex functional $[6,14]$ :

$$
J_{\varepsilon}\left(\phi_{0}\right)=\frac{1}{2} \int_{0}^{T} \int_{\omega}|z|^{2} d x d t+\int_{0}^{T} \int_{\Omega} \mathcal{T} \cdot z d x d t+\varepsilon\left\|\phi_{0}\right\|_{0, \Omega}
$$

Thus, the minimum of $J_{\varepsilon}$ is attained at some $\widehat{\phi}_{0 \varepsilon} \in L^{2}(\Omega)^{2}$. We denote by $\left(\widehat{\phi}_{\varepsilon}, \widehat{\theta}_{\varepsilon}\right)$, $\left(\widehat{z}_{\varepsilon}, \widehat{r}_{\varepsilon}\right)$ the corresponding solution to $(2.1)-(2.2)$ with $\phi_{0}=\widehat{\phi}_{0 \varepsilon}$; then the control function defined as

$$
h_{\varepsilon}=\widehat{z}_{\varepsilon} 1_{\omega}
$$

is such that the associated solution $\left(\bar{u}_{\varepsilon}, \bar{p}_{\varepsilon}\right),\left(q_{\varepsilon}, \pi_{\varepsilon}\right)$ to (1.5)-(1.6) with $u_{0}=0$ satisfies $\left\|q_{\varepsilon}(0)\right\|_{0, \Omega} \leq \varepsilon$.

It is not difficult to see that

$$
\liminf _{\left\|\phi_{0}\right\|_{0, \Omega} \rightarrow \infty} \frac{J_{\varepsilon}\left(\phi_{0}\right)}{\left\|\phi_{0}\right\|_{0, \Omega}} \geq \varepsilon
$$

The proof of this inequality is classical; see [6]. It is implied by the unique continuation property for the cascade adjoint system that we presented above (see Lemma 2.1).

Furthermore, the following optimality condition must be satisfied at $\widehat{\phi}_{0 \varepsilon}$ :

$$
\int_{0}^{T} \int_{\omega}\left|\widehat{z}_{\varepsilon}\right|^{2} d x d t+\int_{0}^{T} \int_{\Omega} \mathcal{T} \cdot \widehat{z}_{\varepsilon} d x d t+\varepsilon\left\|\widehat{\phi}_{0 \varepsilon}\right\|_{0, \Omega}=0
$$

By replacing (3.3) in (3.4), introducing the weight $e^{M t^{-4}}$, and using (3.1) and Young's inequality, we easily deduce that

$$
\int_{0}^{T} \int_{\omega}\left|h_{\varepsilon}\right|^{2} d x d t \leq K^{2} \int_{0}^{T} \int_{\Omega} \exp \left(M t^{-4}\right)|\mathcal{T}|^{2} d x d t
$$

Since $\left\{h_{\varepsilon}\right\}$ is uniformly bounded in $L^{2}(\omega \times(0, T))^{2}$, then up to a subsequence, still denoted $\left\{h_{\varepsilon}\right\}$, we have

$$
\begin{aligned}
& h_{\varepsilon} \rightarrow h \quad \text { weakly in } \quad L^{2}(\omega \times(0, T))^{2} \text {, } \\
& \bar{u}_{\varepsilon} \rightarrow \bar{u} \quad \text { strongly in } L^{2}(Q)^{2}, \quad \text { and } \\
& q_{\varepsilon} \rightarrow q \text { strongly in } L^{2}(Q)^{2},
\end{aligned}
$$

as $\varepsilon \rightarrow 0$. Of course, we have denoted here by $\left(\bar{u}_{\varepsilon}, \bar{p}_{\varepsilon}\right),\left(q_{\varepsilon}, \pi_{\varepsilon}\right)$ and $(\bar{u}, \bar{p}),(q, \pi)$ the solutions to (1.5)-(1.6) associated with $h_{\varepsilon}$ and $h$, respectively. Notice that $\left\|q_{\varepsilon}(0)\right\|_{0, \Omega} \leq \varepsilon$ and consequently we have $q(0)=0$. This ends the proof of Theorem 1.3 .

3.1. A global Carleman estimate. The goal of this section is to present an estimate of the Carleman kind for the solutions to the adjoint cascade system (2.1)-(2.2). As mentioned above, this estimate will be crucial for the proof of Proposition 3.1. 
Let us first introduce an open ball $B_{0}$ such that $B_{0} \subset \subset \omega \cap \mathcal{O}$ and an auxiliary function $\eta_{0} \in \mathcal{C}^{2}(\bar{\Omega})$ satisfying

$$
\eta_{0}(x)>0 \quad \forall x \in \Omega, \quad \eta_{0}=0 \quad \text { on } \partial \Omega, \quad\left|\nabla \eta_{0}(x)\right|>0 \quad \forall x \in \overline{\Omega \backslash B_{0}} .
$$

The existence of such a function is proved in [9].

Let us also introduce the weight functions

$$
\begin{array}{lll}
\alpha(x, t)=\frac{e^{2 \lambda\left\|\eta_{0}\right\|_{\infty}}-e^{\lambda \eta_{0}}}{t^{4}(T-t)^{4}}, & \widehat{\alpha}(t)=\min _{\bar{\Omega}} \alpha(x, t), & \alpha^{*}(t)=\max _{\bar{\Omega}} \alpha(x, t), \\
\varphi(x, t)=\frac{e^{\lambda \eta_{0}}}{t^{4}(T-t)^{4}}, & \widehat{\varphi}(t)=\max _{\bar{\Omega}} \varphi(x, t), & \varphi^{*}(t)=\min _{\bar{\Omega}} \varphi(x, t) .
\end{array}
$$

The following property of the functions $\alpha^{*}$ and $\widehat{\alpha}$ will be needed later.

Lemma 3.2. For any $a>1$ there exists $\lambda_{a}>0$ such that

$$
a \widehat{\alpha}(t)>\alpha^{*}(t) \quad \forall \lambda>\lambda_{a}, \quad \forall t \in(0, T) .
$$

Proof. The proof is elementary. It suffices to notice that we have $a\left(e^{2 x}-e^{x}\right)>$ $e^{2 x}-1$ if $a>1$ and $x$ is sufficiently large.

The main result in this section is the following.

TheOrem 3.3. Assume that $\omega \cap \mathcal{O} \neq \emptyset$ and let the functions $\alpha, \varphi, \widehat{\alpha}$, and $\widehat{\varphi}$ be as above. For each $\widehat{\gamma} \in(0,1)$, there exist constants $\widehat{s}, \widehat{\lambda}$, and $\widehat{C}$ depending on $\Omega, \omega$, $\mathcal{O}, T$, and $\hat{\gamma}$ such that one has

$$
\begin{aligned}
& \int_{0}^{T} \int_{\Omega} e^{-2 s \alpha}\left(\frac{1}{s \varphi}\left(|z|^{2}+|\Delta z|^{2}\right)+s \lambda^{2} \varphi|\nabla z|^{2}+s^{3} \lambda^{4} \varphi^{3}|z|^{2}\right) d x d t \\
& \quad+\int_{0}^{T} \int_{\Omega} e^{-2 s \alpha}\left(\frac{1}{s \varphi}\left(\left|\phi_{t}\right|^{2}+|\Delta \phi|^{2}\right)+s \lambda^{2} \varphi|\nabla \phi|^{2}+s^{3} \lambda^{4} \varphi^{3}|\phi|^{2}\right) d x d t \\
& \quad \leq \widehat{C} \int_{0}^{T} \int_{\omega} e^{-(1+\widehat{\gamma}) s \widehat{\alpha}} s^{63} \lambda^{32} \widehat{\varphi}^{67}|z|^{2} d x d t
\end{aligned}
$$

for any $s>\widehat{s}$ and $\lambda>\widehat{\lambda}$ and for every solution $(\phi, \theta),(z, r)$ to (2.1)-(2.2) associated with initial data $\phi_{0} \in H$.

The proof will be divided in several steps and will be given in the following subsections. First, we will apply a global Carleman estimate for the Stokes system to (2.1) and (2.2). This will lead to the estimate (3.10). Then, to deduce (3.6), we will have to estimate the integral in the right-hand side of (3.10) containing $\phi$ in terms of $z$. To this end, we will follow the steps of the proof of Lemma 2.1 in reverse order.

3.1.1. Step 1: A first direct Carleman estimate. Let $I(s, \lambda ; v)$ stand for the quantity

$$
I(s, \lambda ; v)=\int_{0}^{T} \int_{\Omega} e^{-2 s \alpha}\left(\frac{1}{s \varphi}\left(\left|v_{t}\right|^{2}+|\Delta v|^{2}\right)+s \lambda^{2} \varphi|\nabla v|^{2}+s^{3} \lambda^{4} \varphi^{3}|v|^{2}\right) d x d t
$$

for any positive $s$ and $\lambda$ and any sufficiently regular function $v=v(x, t)$. We then have the following. 
Lemma 3.4. For each $\gamma_{1} \in(0,1)$ there exist positive constants $s_{1}, \lambda_{1}$, and $C_{1}$, depending on $\Omega, \omega, \mathcal{O}, T$, and $\gamma_{1}$, with the following properties:

$$
\begin{aligned}
I(s, \lambda ; z) \leq C_{1}\left\{\int_{0}^{T} \int_{B_{0}} e^{-\left(1+\gamma_{1}\right) s \widehat{\alpha}} s^{7} \lambda^{4} \widehat{\varphi}^{15 / 2}|z|^{2} d x d t\right. \\
+\int_{0}^{T} \int_{B_{0}} e^{-2 s \widehat{\alpha}}(s \lambda \widehat{\varphi})^{2}|\phi|^{2} d x d t \\
\left.+\int_{0}^{T} \int_{\mathcal{O}} e^{-2 s \alpha}\left((s \varphi)^{1 / 2}|\phi|^{2}+\frac{1}{s^{3} \varphi^{7 / 2}}\left|\phi_{t}\right|^{2}\right) d x d t\right\}
\end{aligned}
$$

and

$$
I(s, \lambda ; \phi) \leq C_{1} \int_{0}^{T} \int_{B_{0}} e^{-\left(1+\gamma_{1}\right) s \widehat{\alpha}} s^{7} \lambda^{4} \widehat{\varphi}^{15 / 2}|\phi|^{2} d x d t
$$

for any $s>s_{1}$ and $\lambda>\lambda_{1}$ and for every solution of (2.1)-(2.2) with $\phi_{0} \in H$.

The proof of Lemma 3.4 is similar to the proof of other recent global Carleman inequalities for the Stokes system. The main ideas are due to Imanuvilov [10, 11]; also see [8] for other related results. The proof is presented in the appendix.

Let us fix $\hat{\gamma}$, with $0<\widehat{\gamma}<1$. We are now going to deduce several estimates that hold for "sufficiently large $s$ and $\lambda . "$ By this we mean that they are satisfied for any $s>\bar{s}$ and any $\lambda>\bar{\lambda}$, where $\bar{s}$ and $\bar{\lambda}$ are (large) positive constants depending only on $\Omega, \omega, \mathcal{O}, T$, and $\widehat{\gamma}$.

In what follows, $C$ denotes a generic constant, not necessarily the same at each occurrence, depending on $\Omega, \omega, \mathcal{O}, T$, and (possibly) $\widehat{\gamma}$.

Let $\gamma_{1}$ be given in $(\widehat{\gamma}, 1)$. In view of Lemma 3.4 applied to $\gamma_{1}$, we get

$$
I(s, \lambda ; z)+I(s, \lambda ; \phi) \leq C \int_{0}^{T} \int_{B_{0}} e^{-\left(1+\gamma_{1}\right) s \widehat{\alpha}} s^{7} \lambda^{4} \widehat{\varphi}^{15 / 2}\left(|z|^{2}+|\phi|^{2}\right) d x d t
$$

for $s$ and $\lambda$ large enough.

Indeed, the last two integrals in (3.8) can be absorbed by the left-hand side of $I(s, \lambda ; \phi)$, since

$$
C s^{-3} \varphi^{-7 / 2} \leq \frac{1}{2}(s \varphi)^{-1} \quad \text { and } \quad C(s \varphi)^{1 / 2} \leq \frac{1}{2} s^{3} \varphi^{3}
$$

for sufficiently large $s$.

3.1.2. Step 2: An estimate of $\phi$ in terms of $\operatorname{curl} \phi$. To simplify the notation, let us set $a=7$ and $b=15 / 2$. Then

$$
I(s, \lambda ; z)+I(s, \lambda ; \phi) \leq C \int_{0}^{T} \int_{B_{0}} e^{-\left(1+\gamma_{1}\right) s \widehat{\alpha}} s^{a} \lambda^{4} \widehat{\varphi}^{b}\left(|z|^{2}+|\phi|^{2}\right) d x d t .
$$

We will denote by $B_{1}, B_{2}, \ldots$ a sequence of balls centered at the same point as $B_{0}$ and satisfying

$$
B_{0} \subset \subset B_{1} \subset \subset \cdots \subset \subset \omega \cap \mathcal{O} .
$$


It is not a restriction to assume that their common center is the origin. This will be supposed in what follows for simplicity. We will consider some functions $\xi_{i} \in \mathcal{C}_{0}^{\infty}\left(B_{i}\right)$ satisfying

$$
\begin{gathered}
0 \leq \xi_{i} \leq 1, \quad \xi_{i}(x)=1 \text { in } B_{i-1}, \\
\xi_{i}^{-1 / 2} \nabla \xi_{i} \in L^{\infty}(\Omega), \quad \xi_{i}^{-1 / 2} \Delta \xi_{i} \in L^{\infty}(\Omega) .
\end{gathered}
$$

(See [18] for a justification of the existence of these $\xi_{i}$.)

Since $\operatorname{div} \phi=0, \phi=0$ on $\Sigma$, and $\Omega$ is connected, we can introduce the stream function $\psi$ satisfying

$$
\phi=\operatorname{curl} \psi \equiv\left(\frac{\partial \psi}{\partial x_{2}},-\frac{\partial \psi}{\partial x_{1}}\right)
$$

with $\psi=0$ on one connected component of $\Sigma$ and $\frac{\partial \psi}{\partial n}=0$ on $\Sigma$.

Let us set $\rho_{1}(t)=e^{-\left(1+\gamma_{1}\right) s \widehat{\alpha}} s^{a} \lambda^{4} \widehat{\varphi}^{b}$. Then we have

$$
\int_{0}^{T} \int_{B_{0}} \rho_{1}|\phi|^{2} d x d t \leq \int_{0}^{T} \int_{B_{1}} \rho_{1} \xi_{1}|\nabla \psi|^{2} d x d t .
$$

We will now give an estimate of the last integral in terms of $|\operatorname{curl} \phi|^{2}$. To this end, let us introduce the vorticity $w$, given by

$$
w=\operatorname{curl} \phi=\frac{\partial \phi_{2}}{\partial x_{1}}-\frac{\partial \phi_{1}}{\partial x_{2}} .
$$

Applying the curl operator to (2.1), we obtain

$$
\left\{\begin{array}{l}
w_{t}-\Delta w+w-\frac{\partial \psi}{\partial x_{1}}=0 \quad \text { in } Q \\
\Delta \psi+w=0 \quad \text { in } Q .
\end{array}\right.
$$

To estimate $|\nabla \psi|^{2}$, we multiply by $\rho_{1} \xi_{1} \psi$ the second equation of (3.13). Then, we integrate by parts with respect to the space variable $x$ and we get

$$
\int_{0}^{T} \int_{B_{1}} \rho_{1} \xi_{1}|\nabla \psi|^{2} d x d t=\int_{0}^{T} \int_{B_{1}} \rho_{1} \xi_{1} \psi w d x d t+\frac{1}{2} \int_{0}^{T} \int_{B_{1}} \rho_{1}\left(\Delta \xi_{1}\right)|\psi|^{2} d x d t
$$

Notice that using $I(s, \lambda ; \phi)$, we can get upper bounds for $|\psi|^{2},|\nabla \psi|^{2}$, and $\left|\psi_{t}\right|^{2}$. Indeed, from the definition of $\alpha^{*}, \varphi^{*}$, and $\widehat{\varphi}$, we have

$$
\begin{aligned}
I(s, \lambda ; \phi) & \geq \int_{0}^{T} \int_{\Omega} e^{-2 s \alpha}\left(\frac{1}{s \varphi}\left|\nabla \psi_{t}\right|^{2}+s^{3} \lambda^{4} \varphi^{3}|\nabla \psi|^{2}\right) d x d t \\
& \geq \int_{0}^{T} \int_{\Omega} e^{-2 s \alpha^{*}}\left(\frac{1}{s \widehat{\varphi}}\left|\nabla \psi_{t}\right|^{2}+s^{3} \lambda^{4}\left(\varphi^{*}\right)^{3}|\nabla \psi|^{2}\right) d x d t \\
& \geq C \int_{0}^{T} \int_{\Omega} e^{-2 s \alpha^{*}}\left(\frac{1}{s \widehat{\varphi}}\left|\psi_{t}\right|^{2}+s^{3} \lambda^{4}\left(\varphi^{*}\right)^{3}\left(|\psi|^{2}+|\nabla \psi|^{2}\right)\right) d x d t .
\end{aligned}
$$

Here we have used the fact that $\psi=0$ on one of the connected components of $\Sigma$ to apply Poincaré's inequality. 
With this information, we will be able to absorb the first integral in (3.14). Indeed, after using Young's inequality, we can estimate this term as follows:

$$
\begin{aligned}
& \int_{0}^{T} \int_{B_{1}} \rho_{1} \xi_{1} \psi w d x d t \leq \delta \int_{0}^{T} \int_{\Omega} e^{-2 s \alpha^{*}} s^{3} \lambda^{4}\left(\varphi^{*}\right)^{3}|\psi|^{2} d x d t \\
& \quad+C_{\delta} \int_{0}^{T} \int_{B_{1}} e^{-2\left(1+\gamma_{1}\right) s \widehat{\alpha}+2 s \alpha^{*}} s^{2 a-3} \lambda^{4} \widehat{\varphi}^{2 b-3}|w|^{2} d x d t .
\end{aligned}
$$

Now, if we introduce $\gamma_{2}$ with $0<\gamma_{2}<2 \gamma_{1}-1$, then $\left(1+2 \gamma_{1}-\gamma_{2}\right) / 2>1$ and, from Lemma 3.2 , we see that $\left(1+2 \gamma_{1}-\gamma_{2}\right) \widehat{\alpha} / 2>\alpha^{*}$ for $\lambda$ sufficiently large. Consequently, it can be assumed that

$$
-2\left(1+\gamma_{1}\right) \widehat{\alpha}+2 \alpha^{*}<-\left(1+\gamma_{2}\right) \widehat{\alpha}
$$

and we can replace $e^{-2\left(1+\gamma_{1}\right) s \widehat{\alpha}+2 s \alpha^{*}}$ by $e^{-\left(1+\gamma_{2}\right) s \widehat{\alpha}}$ in the last integral in (3.16):

$$
\begin{gathered}
\int_{0}^{T} \int_{B_{1}} \rho_{1} \xi_{1} \psi w d x d t \leq \delta \int_{0}^{T} \int_{\Omega} e^{-2 s \alpha^{*}} s^{3} \lambda^{4}\left(\varphi^{*}\right)^{3}|\psi|^{2} d x d t \\
+C_{\delta} \int_{0}^{T} \int_{B_{1}} e^{-\left(1+\gamma_{2}\right) s \widehat{\alpha}} s^{2 a-3} \lambda^{4} \widehat{\varphi}^{2 b-3}|w|^{2} d x d t .
\end{gathered}
$$

Notice that if we had chosen $\gamma_{1}$ sufficiently close to 1 before, then we would still have the possibility of choosing $\gamma_{2}$ satisfying $\widehat{\gamma}<\gamma_{2}<2 \gamma_{1}-1$.

On the other hand, by choosing $\delta$ sufficiently small, we can absorb the first term in the right-hand side of $(3.17)$ with $I(s, \lambda ; \phi)$.

It remains in this step to estimate the last integral in (3.14). Assume that $\xi_{1}$ has been constructed as before but also satisfying

$$
\xi_{1}(x)= \begin{cases}1 & \text { in }|x|<r_{0}, \\ \widehat{\Psi}\left(\frac{|x|-r_{0}}{r_{1}-a-r_{0}}\right) & \text { in } r_{0} \leq|x| \leq r_{1}-a, \\ 0 & \text { in }|x|>r_{1}-a,\end{cases}
$$

where $r_{i}$ denotes the radius of $B_{i}, a$ is small enough, and $\widehat{\Psi}$ is a function satisfying $\widehat{\Psi} \in \mathcal{C}^{\infty}([0,1])$

$$
\widehat{\Psi}(0)=1, \quad \widehat{\Psi}(1)=0, \quad \text { and } \quad \widehat{\Psi}^{(n)}(0)=\widehat{\Psi}^{(n)}(1)=0 \quad \forall n \geq 1 .
$$

Let us set

$$
\eta(x)=\int_{\bar{x}_{1}}^{x_{1}} \Delta \xi_{1}\left(y_{1}, x_{2}\right) d y_{1}
$$

where for each $x=\left(x_{1}, x_{2}\right) \in \bar{B}_{1}$ we take $\bar{x}_{1}<x_{1}$ and $\left(\bar{x}_{1}, x_{2}\right) \in \partial B_{1}$. Notice that $\frac{\partial \eta}{\partial x_{1}}=\Delta \xi_{1}$. It is also easy to see that Supp $\eta \subset \bar{B}_{1}\left(0 ; r_{1}-a\right)$. And now, using the first equation in (3.13), we observe that

$$
\begin{aligned}
\frac{1}{2} \int_{0}^{T} \int_{B_{1}} \rho_{1}\left(\Delta \xi_{1}\right)|\psi|^{2} d x d t & =\frac{1}{2} \int_{0}^{T} \int_{B_{1}} \rho_{1} \frac{\partial \eta}{\partial x_{1}}|\psi|^{2} d x d t \\
& =-\int_{0}^{T} \int_{B_{1}} \rho_{1} \eta \psi \frac{\partial \psi}{\partial x_{1}} d x d t \\
& =-\int_{0}^{T} \int_{B_{1}} \rho_{1} \eta \psi\left(w_{t}-\Delta w+w\right) d x d t .
\end{aligned}
$$


Remark 4. Notice that we used the term $\frac{\partial \psi}{\partial x_{1}}$ in equation (3.13) to estimate $|\psi|^{2}$ over $B_{1}$. The term comes from Coriolis force and it is absent in the Stokes system.

We will now estimate this last integral in the right-hand side of (3.18). Concerning the product $\rho_{1} \eta \psi w_{t}$, we can integrate by parts with respect to time in $B_{1} \times(0, T)$ and then apply Young's inequality to deduce that

$$
\begin{aligned}
\int_{0}^{T} \int_{B_{1}} \rho_{1} \eta \psi w_{t} d x d t & =-\int_{0}^{T} \int_{B_{1}}\left(\rho_{1} \eta \psi_{t} w+\rho_{1}^{\prime} \eta \psi w\right) d x d t \\
& \leq \delta \int_{0}^{T} \int_{\Omega} e^{-2 s \alpha^{*}}\left(\frac{1}{s \widehat{\varphi}}\left|\psi_{t}\right|^{2}+s^{3} \lambda^{4}\left(\varphi^{*}\right)^{3}|\psi|^{2}\right) d x d t \\
& +C_{\delta} \int_{0}^{T} \int_{B_{1}} e^{-\left(1+\gamma_{2}\right) s \widehat{\alpha}}\left(s^{2 a+1} \lambda^{8} \widehat{\varphi}^{2 b+1}+s^{2 a-1} \lambda^{4} \widehat{\varphi}^{2 b-1 / 2}\right)|w|^{2} d x d t
\end{aligned}
$$

for sufficiently large $s$ and $\lambda$.

To obtain this inequality, we first used that

$$
\left|\rho_{1}^{\prime}\right|=\left|\left(e^{-\left(1+\gamma_{1}\right) s \widehat{\alpha}} s^{a} \lambda^{4} \widehat{\varphi}^{b}\right) t\right| \leq C e^{-\left(1+\gamma_{1}\right) s \widehat{\alpha}} s^{a+1} \lambda^{4} \widehat{\varphi}^{b+5 / 4} .
$$

Then, we noticed that

$$
\begin{aligned}
& \int_{0}^{T} \int_{B_{1}} \rho_{1}^{\prime} \eta \psi w d x d t \\
& \quad \leq \delta \int_{0}^{T} \int_{\Omega} e^{-2 s \alpha^{*}} s^{3} \lambda^{4}\left(\varphi^{*}\right)^{3}|\psi|^{2} d x d t \\
& \quad+C_{\delta} \int_{0}^{T} \int_{B_{1}} e^{-2\left(1+\gamma_{1}\right) s \widehat{\alpha}+2 s \alpha^{*}} s^{2 a-1} \lambda^{4} \widehat{\varphi}^{2 b+5 / 2}\left(\varphi^{*}\right)^{-3}|w|^{2} d x d t
\end{aligned}
$$

and, finally, we took $s$ and $\lambda$ large enough to have

$$
e^{-2\left(1+\gamma_{1}\right) s \widehat{\alpha}+2 s \alpha^{*}} \widehat{\varphi}^{2 b+5 / 2}\left(\varphi^{*}\right)^{-3} \leq e^{-\left(1+\gamma_{2}\right) s \widehat{\alpha}} \widehat{\varphi}^{2 b-1 / 2} .
$$

We can simplify the estimate (3.19) by using the inequality

$$
s^{2 a-1} \widehat{\varphi}^{2 b-1 / 2} \leq C s^{2 a+1} \widehat{\varphi}^{2 b+1},
$$

which must hold for large $s$. Thus, we obtain

$$
\begin{aligned}
& \int_{0}^{T} \int_{B_{1}} \rho_{1} \eta \psi w_{t} d x d t \\
& \quad \leq \delta \int_{0}^{T} \int_{\Omega} e^{-2 s \alpha^{*}}\left(\frac{1}{s \widehat{\varphi}}\left|\psi_{t}\right|^{2}+s^{3} \lambda^{4}\left(\varphi^{*}\right)^{3}|\psi|^{2}\right) d x d t \\
& \quad+C_{\delta} \int_{0}^{T} \int_{B_{1}} e^{-\left(1+\gamma_{2}\right) s \widehat{\alpha}} s^{2 a+1} \lambda^{8} \widehat{\varphi}^{2 b+1}|w|^{2} d x d t .
\end{aligned}
$$

Notice that the first integral in the right-hand side of (3.20) also appears in (3.15) and can be absorbed later by choosing $\delta$ small enough.

Let us now consider the term $\rho_{1} \eta \psi(\Delta w)$ in the last integral of (3.18). Let us integrate by parts with respect to the space variable $x$, let us use the identity $\Delta \psi=w$, 
and let us apply Young's inequality. Arguing as before, we obtain

$$
\begin{aligned}
\int_{0}^{T} \int_{B_{1}} \rho_{1} \eta \psi(\Delta w) d x d t & =\int_{0}^{T} \int_{B_{1}} \rho_{1}\left((\Delta \eta) \psi w+2 \nabla \eta \cdot \nabla \psi w+\eta|w|^{2}\right) d x d t \\
& \leq \delta \int_{0}^{T} \int_{\Omega} e^{-2 s \alpha^{*}} s^{3} \lambda^{4}\left(\varphi^{*}\right)^{3}\left(|\psi|^{2}+|\nabla \psi|^{2}\right) d x d t \\
& +C_{\delta} \int_{0}^{T} \int_{B_{1}} e^{-\left(1+\gamma_{2}\right) s \widehat{\alpha}} s^{2 a-3} \lambda^{4} \widehat{\varphi}^{2 b-3}|w|^{2} d x d t
\end{aligned}
$$

for any sufficiently large $s$ and $\lambda$.

Finally, arguing in a similar way, we can also estimate the last term $\rho_{1} \eta \psi w$ in

$$
\begin{aligned}
\int_{0}^{T} \int_{B_{1}} \rho_{1} \eta \psi w d x d t & \leq \delta \int_{0}^{T} \int_{B_{1}} e^{-2 s \alpha^{*}} s^{3} \lambda^{4}\left(\varphi^{*}\right)^{3}|\psi|^{2} d x d t \\
& +C_{\delta} \int_{0}^{T} \int_{B_{1}} e^{-\left(1+\gamma_{2}\right) s \widehat{\alpha}} s^{2 a-3} \lambda^{4} \widehat{\varphi}^{2 b-3}|w|^{2} d x d t
\end{aligned}
$$

From (3.18) and (3.20)-(3.22), we find that

$$
\begin{aligned}
\frac{1}{2} \int_{0}^{T} \int_{B_{1}} \rho_{1}\left(\Delta \xi_{1}\right)|\psi|^{2} d x d t & \\
& \leq 3 \delta \int_{0}^{T} \int_{\Omega} e^{-2 s \alpha^{*}}\left(\frac{1}{s \widehat{\varphi}}\left|\psi_{t}\right|^{2}+s^{3} \lambda^{4}\left(\varphi^{*}\right)^{3}\left(|\psi|^{2}+|\nabla \psi|^{2}\right)\right) d x d t \\
& +C_{\delta} \int_{0}^{T} \int_{B_{1}} \rho_{2}|w|^{2} d x d t
\end{aligned}
$$

where

$$
\rho_{2}(t)=e^{-\left(1+\gamma_{2}\right) s \widehat{\alpha}} s^{2 a+1} \lambda^{8} \widehat{\varphi}^{2 b+1} .
$$

Replacing the estimates (3.16) and (3.23) in (3.10), with $\delta>0$ sufficiently small, we obtain

$(3.24) I(s, \lambda ; z)+I(s, \lambda ; \phi) \leq C\left\{\int_{0}^{T} \int_{B_{0}} \rho_{1}|z|^{2} d x d t+\int_{0}^{T} \int_{B_{1}} \rho_{2}|\operatorname{curl} \phi|^{2} d x d t\right\}$.

3.1.3. Step 3: An estimate of $\operatorname{curl} \phi$ in terms of $z$. Let us apply the curl operator to $(2.2)$. For $\zeta=\operatorname{curl} z$, we obtain the following:

$$
-\zeta_{t}-\Delta \zeta+\zeta-z_{2}=w 1_{\mathcal{O}} \quad \text { in } \mathcal{O} \times(0, T)
$$

Recall that $\xi_{2} \in \mathcal{C}_{0}^{\infty}\left(B_{2}\right)$ satisfies (3.12) and $B_{1} \subset \subset B_{2} \subset \subset \omega \cap \mathcal{O}$. After multiplying the above equation by $\rho_{2} \xi_{2} w$, integrating by parts in $Q$, and using (3.13), it follows that

$$
\begin{aligned}
\int_{0}^{T} \int_{B_{2}} \rho_{2} \xi_{2}|w|^{2} d x d t= & -\int_{0}^{T} \int_{B_{2}} \rho_{2} \xi_{2} \phi_{2} \zeta d x d t+\int_{0}^{T} \int_{B_{2}} \rho_{2}^{\prime} \xi_{2} w \zeta d x d t \\
& -\int_{0}^{T} \int_{B_{2}} \rho_{2}\left(\left(\Delta \xi_{2}\right) w \zeta+2\left(\nabla \xi_{2} \cdot \nabla w\right) \zeta+\xi_{2} w z_{2}\right) d x d t
\end{aligned}
$$


As before, we choose $\gamma_{3}$ satisfying $0<\gamma_{3}<2 \gamma_{2}-1$. Then, for sufficiently large $\lambda$ we have $\left(1+2 \gamma_{2}-\gamma_{3}\right) \widehat{\alpha} / 2>\alpha^{*}$ and, consequently,

$$
-2\left(1+\gamma_{2}\right) \widehat{\alpha}+2 s \alpha<-2\left(1+\gamma_{2}\right) \widehat{\alpha}+2 s \alpha^{*}<-\left(1+\gamma_{3}\right) \widehat{\alpha} .
$$

Notice once more that if $\gamma_{1}$ is sufficiently close to 1 , then we can choose $\gamma_{3}$ in $(\widehat{\gamma}, 1)$.

Now, proceeding as in the previous step, we see that

$$
\begin{aligned}
\left|\int_{0}^{T} \int_{B_{2}} \rho_{2} \xi_{2} \phi_{2} \zeta d x d t\right| & \leq \delta \int_{0}^{T} \int_{\Omega} e^{-2 s \alpha} s^{3} \lambda^{4} \varphi^{3}\left|\phi_{2}\right|^{2} d x d t \\
& +C_{\delta} \int_{0}^{T} \int_{B_{2}} \rho_{3} \frac{1}{s^{4} \lambda^{4} \widehat{\varphi}^{4}} \xi_{2}^{2}|\zeta|^{2} d x d t
\end{aligned}
$$

for any small $\delta>0$ (to be fixed later). Here, $\rho_{3}$ stands for the function

$$
\rho_{3}(t)=e^{-\left(1+\gamma_{3}\right) s \widehat{\alpha}(t)} s^{4 a+3} \lambda^{16} \widehat{\varphi}^{4 b+3}(t) .
$$

We also have

$$
\begin{aligned}
\int_{0}^{T} \int_{B_{2}} \rho_{2}^{\prime} \xi_{2} w \zeta d x d t & \leq \delta \int_{0}^{T} \int_{B_{2}} \rho_{2} \xi_{2}|w|^{2} d x d t \\
& +C_{\delta} \int_{0}^{T} \int_{B_{2}} \rho_{2} s^{2} \widehat{\varphi}^{5 / 2} \xi_{2}|\zeta|^{2} d x d t
\end{aligned}
$$

Furthermore, after separating the terms in the last integral in (3.25), we find that

$$
\begin{aligned}
\left|\int_{0}^{T} \int_{B_{2}} \rho_{2}\left(\Delta \xi_{2}\right) w \zeta d x d t\right| & \leq \delta \int_{0}^{T} \int_{B_{2}} \rho_{2} \xi_{2}|w|^{2} d x d t \\
& +C_{\delta} \int_{0}^{T} \int_{B_{2}} \rho_{2} \frac{\left|\Delta \xi_{2}\right|^{2}}{\xi_{2}}|\zeta|^{2} d x d t
\end{aligned}
$$

and

$$
\begin{aligned}
\left|\int_{0}^{T} \int_{B_{2}} \rho_{2}\left(\nabla \xi_{2} \cdot \nabla w\right) \zeta d x d t\right| & \leq \delta \int_{0}^{T} \int_{\Omega} e^{-2 s \alpha} \frac{1}{s \varphi}|\Delta \phi|^{2} d x d t \\
& +C_{\delta} \int_{0}^{T} \int_{B_{2}} \rho_{3}\left|\nabla \xi_{2}\right|^{2}|\zeta|^{2} d x d t .
\end{aligned}
$$

In this last estimate we have used that $|\nabla w|^{2}=|\Delta \phi|^{2}$. Finally,

$$
\left|\int_{0}^{T} \int_{B_{2}} \rho_{2} \xi_{2} w z_{2} d x d t\right| \leq \delta \int_{0}^{T} \int_{B_{2}} \rho_{2} \xi_{2}|w|^{2} d x d t+C_{\delta} \int_{0}^{T} \int_{B_{2}} \rho_{2} \xi_{2}\left|z_{2}\right|^{2} d x d t .
$$

In view of (3.25) and all these inequalities, we obtain

$$
\begin{aligned}
\int_{0}^{T} \int_{B_{2}} \rho_{2} \xi_{2}|\operatorname{curl} \phi|^{2} d x d t \\
\leq \\
\leq \frac{\delta}{1-3 \delta} \int_{0}^{T} \int_{\Omega} e^{-2 s \alpha}\left(\frac{1}{s \varphi}|\Delta \phi|^{2}+s^{3} \lambda^{4} \varphi^{3}\left|\phi_{2}\right|^{2}\right) d x d t \\
\quad+C_{\delta} \int_{0}^{T} \int_{B_{2}}\left(\rho_{2}\left|z_{2}\right|^{2}+\rho_{3} \bar{\xi}_{2}|\operatorname{curl} z|^{2}\right) d x d t
\end{aligned}
$$

for some $\bar{\xi}_{2} \in \mathcal{C}_{0}^{\infty}\left(B_{2}\right)$. 
It remains to estimate the previous integral of $\rho_{3} \bar{\xi}_{2}|\operatorname{curl} z|^{2}$. Arguing as above, we see that

$$
\begin{aligned}
\int_{0}^{T} \int_{B_{2}} \rho_{3} \bar{\xi}_{2}|\operatorname{curl} z|^{2} d x d t & \leq 2 \int_{0}^{T} \int_{B_{2}} \rho_{3} \bar{\xi}_{2}|\nabla z|^{2} d x d t \\
& =-2 \int_{0}^{T} \int_{B_{2}} \rho_{3}\left(\nabla \bar{\xi}_{2} \cdot \nabla z+\bar{\xi}_{2}(\Delta z)\right) z d x d t \\
& \leq \delta \int_{0}^{T} \int_{\Omega} e^{-2 s \alpha}\left(s \lambda^{2} \varphi|\nabla z|^{2}+\frac{1}{s \varphi}|\Delta z|^{2}\right) d x d t \\
& +C_{\delta} \int_{0}^{T} \int_{B_{2}} \rho_{4}|z|^{2} d x d t
\end{aligned}
$$

where

$$
\rho_{4}(t)=e^{-\left(1+\gamma_{4}\right) s \widehat{\alpha}} s^{8 a+7} \lambda^{32} \widehat{\varphi}^{8 b+7}
$$

for some $\gamma_{4}$ satisfying $0<\gamma_{4}<2 \gamma_{3}-1$. For the reasons stated above, it is clear that $\gamma_{4}$ can be assumed to satisfy $\widehat{\gamma}<\gamma_{4}<1$.

Choosing $\delta>0$ small enough and replacing the estimates (3.26) and (3.27) in (3.24), we obtain

$$
I(s, \lambda ; z)+I(s, \lambda ; \phi) \leq C \int_{0}^{T} \int_{\omega} \rho_{4}|z|^{2} d x d t
$$

for all large $s$ and $\lambda$. Taking into account the definition of $\rho_{4}$, that $\gamma_{4}>\widehat{\gamma}, a=7$, and $b=15 / 2$, we see that (3.6) holds.

This ends the proof of Theorem 3.3.

3.2. Proof of Proposition 3.1. Let us now give the proof of the observability inequality (3.1) for solutions of system (2.1)-(2.2), which relies on the above result. First, we observe that from the continuous dependence of the solution of (2.2), we have

$$
\int_{T / 2}^{T} \int_{\Omega}|z|^{2} d x d t \leq C \int_{T / 2}^{T} \int_{\mathcal{O}}|\phi|^{2} d x d t
$$

and from classical energy estimates for system (2.1), using the fact that $(k \wedge \phi) \cdot \phi=0$, we obtain an energy decreasing property:

$$
\|\phi(t+T / 4)\|_{0, \Omega}^{2} \leq C\|\phi(t)\|_{0, \Omega}^{2} \quad \forall t \in(T / 4,3 T / 4) .
$$

If we integrate the last inequality over the time interval $(T / 4,3 T / 4)$ and change the integral variable $t \rightarrow t+T / 4$ on the left-hand integral, we can easily deduce that

$$
\int_{T / 2}^{T} \int_{\Omega}|\phi|^{2} d x d t \leq C \int_{T / 4}^{3 T / 4} \int_{\Omega}|\phi|^{2} d x d t
$$

where $C$ is independent of $\phi$.

In what follows, we will fix $s, \lambda$, and $\widehat{\gamma}$ as in Theorem 3.3, depending on $\Omega, \omega, \mathcal{O}$, and $T$, such that (3.6) is satisfied. 
Let us first prove that there exist positive constants $M, C_{1}$ such that

$$
\int_{0}^{T / 2} \int_{\Omega} \exp \left(-M t^{-4}\right)|z|^{2} d x d t+\int_{T / 2}^{T} \int_{\Omega}|\phi|^{2} d x d t \leq C_{1} \int_{0}^{T} \int_{\omega}|z|^{2} d x d t
$$

For this estimate, let us first notice that for some constants $M$ and $C$, one has

$$
e^{-2 s \alpha(x, t)} \varphi(x, t)^{3} \geq C e^{-M t^{-4}} \quad \forall(x, t) \in \bar{\Omega} \times(0, T / 2) ;
$$

this is easy to see, in view of the definitions of $\alpha$ and $\varphi$. Now, using (3.6), we get

$$
\begin{array}{r}
\int_{0}^{T / 2} \int_{\Omega} \exp \left(-M t^{-4}\right)|z|^{2} d x d t \leq \frac{1}{s^{3} \lambda^{4}} I(s, \lambda ; z) \\
\leq C K \int_{0}^{T} \int_{\omega} e^{-(1+\widehat{\gamma}) s \widehat{\alpha}} s^{63} \widehat{\varphi}^{67}|z|^{2} d x d t,
\end{array}
$$

and since the weight $e^{-(1+\widehat{\gamma}) s \widehat{\alpha}} \widehat{\varphi}^{67}$ is bounded, we can estimate the first term in the left-hand side of (3.31). On the other hand, to obtain an estimate of $\phi$ in terms of $z$, let us recall the inequality (3.6). Since $e^{-2 s \alpha} t^{-12}(T-t)^{-12}$ is bounded from below far from $t=0$ and $t=T$, in view of (3.30), we have

$$
\begin{aligned}
\int_{T / 2}^{T} \int_{\Omega}|\phi|^{2} d x d t & \leq \int_{T / 4}^{3 T / 4} \int_{\Omega}|\phi|^{2} d x d t \\
& \leq C \int_{T / 4}^{3 T / 4} \int_{\Omega} e^{-2 s \alpha} s^{3} \lambda^{4} \varphi^{3}|\phi|^{2} d x d t \leq C \int_{0}^{T} \int_{\omega} e^{-(1+\widehat{\gamma}) s \widehat{\alpha}} \widehat{\varphi}^{67}|z|^{2} d x d t .
\end{aligned}
$$

As before, from the fact that $e^{-(1+\hat{\gamma}) s \widehat{\alpha}} \widehat{\varphi}^{67}$ is bounded, we are able to estimate the second term in the left-hand side of (3.31).

Finally, the desired observability inequality (3.1) is obtained using the energy estimate (3.29) and (3.31):

$$
\begin{aligned}
\int_{0}^{T} \int_{\Omega} \exp \left(-M t^{-4}\right)|z|^{2} d x d t & \leq \int_{0}^{T / 2} \int_{\Omega} \exp \left(-M t^{-4}\right)|z|^{2} d x d t+\int_{T / 2}^{T} \int_{\Omega}|z|^{2} d x d t \\
& \leq C\left(\int_{0}^{T / 2} \int_{\Omega} \exp \left(-M t^{-4}\right)|z|^{2} d x d t+\int_{T / 2}^{T} \int_{\Omega}|\phi|^{2} d x d t\right) \\
& \leq C \int_{0}^{T} \int_{\omega}|z|^{2} d x d t .
\end{aligned}
$$

Appendix. Proof of Lemma 3.4. Let us recall that this proof is given for the sake of completeness, but it is essentially an adaptation to our framework of the arguments presented in [8] and [11]. Let us consider the system

$$
\left\{\begin{array}{l}
-z_{t}-\Delta z+z-\left(1+x_{2}\right) \mathrm{k} \wedge z+\nabla r=\phi 1_{\mathcal{O}} \text { in } Q, \\
\operatorname{div} z=0 \text { in } Q \\
z=0 \text { on } \Sigma \\
z(T)=0 \text { in } \Omega,
\end{array}\right.
$$

where $\phi \in L^{2}(0, T ; W) \cap H^{1}(0, T ; H)$. 
Recall that $B_{0}$ is an open ball satisfying $B_{0} \subset \subset \omega \cap \mathcal{O}$ and the auxiliary function $\eta_{0}$ satisfies $\eta_{0} \in \mathcal{C}^{2}(\bar{\Omega})$,

$$
\eta_{0}(x)>0 \quad \forall x \in \Omega, \quad \eta_{0}=0 \quad \text { on } \partial \Omega, \quad\left|\nabla \eta_{0}(x)\right|>0 \quad \forall x \in \overline{\Omega \backslash B_{0}} .
$$

We will need an additional open ball $B_{00} \subset \subset B_{0}$, such that we still have

$$
\left|\nabla \eta_{0}(x)\right|>0 \quad \forall x \in \overline{\Omega \backslash B_{00}} .
$$

We will divide the proof of Lemma 3.4 into several steps.

Step 1. Following [8], we apply some well-known Carleman estimates for the heat equation to (3.32). Thus, there exist constants $s_{0}, \lambda_{0}$, and $C>0$ depending on $\Omega$, $\omega$, and $T$ such that for every $\lambda>\lambda_{0}$ and $s>s_{0}$, the following estimate holds:

$$
\begin{aligned}
I(s, \lambda ; z) \leq C & \left\{\int_{0}^{T} \int_{B_{00}} e^{-2 s \alpha} s^{3} \lambda^{4} \varphi^{3}|z|^{2} d x d t\right. \\
& \left.+\int_{0}^{T} \int_{\Omega} e^{-2 s \alpha}\left(|\nabla r|^{2}+\left|\left(1+x_{2}\right) k \wedge z\right|^{2}+\left|\phi 1_{\mathcal{O}}\right|^{2}\right) d x d t\right\}
\end{aligned}
$$

Recall that the definitions of $I(s, \lambda ; z)$ and the weights $\alpha$ and $\varphi$ are given in section 3.1.

Of course, we can choose $s$ large enough to absorb the previous term $\left|\left(1+x_{2}\right) k \wedge z\right|^{2}$ with the left-hand side (3.33). We then have

$$
\begin{aligned}
I(s, \lambda ; z) \leq C & \left\{\int_{0}^{T} \int_{B_{00}} e^{-2 s \alpha} s^{3} \lambda^{4} \varphi^{3}|z|^{2} d x d t\right. \\
& \left.+\int_{0}^{T} \int_{\Omega} e^{-2 s \alpha}|\nabla r|^{2} d x d t+\int_{0}^{T} \int_{\mathcal{O}} e^{-2 s \alpha}|\phi|^{2} d x d t\right\}
\end{aligned}
$$

for any $\lambda>\lambda_{0}$ and any $s>s_{01}$.

Step 2. To estimate the pressure gradient $\nabla r$ in (3.34), we first apply the divergence operator to (3.32), i.e., we write

$$
\Delta r(t)=\operatorname{div}\left(\left(1+x_{2}\right) \mathrm{k} \wedge z\right)(t) \quad \text { in } \Omega, \quad t \in(0, T),
$$

and then we use the following result by Imanuvilov and Puel [12], which is satisfied by weak solutions to second order elliptic equations.

LemmA 3.5. Let us set $\beta(x)=e^{\lambda \eta_{0}(x)}$ and let $v \in H^{1}(\Omega)$ be a solution of

$$
\Delta v=\operatorname{div} h \quad \text { in } \Omega,
$$

where $h \in L^{2}(\Omega)^{2}$. Then there exist positive constants $\tau_{2}, \lambda_{01}$, and $C$ such that

$$
\begin{aligned}
\int_{\Omega} e^{2 \tau \beta}|\nabla v|^{2} d x \leq C & \left\{\tau \int_{\Omega} e^{2 \tau \beta} \beta|h|^{2} d x+\tau^{1 / 2} e^{2 \tau}\|g\|_{1 / 2, \partial \Omega}^{2}\right. \\
& \left.+\tau^{2} \lambda^{2} \int_{B_{00}} e^{2 \tau \beta} \beta^{2}|v|^{2} d x+\int_{B_{00}} e^{2 \tau \beta}|\nabla v|^{2} d x\right\}
\end{aligned}
$$

for any $\tau>\tau_{2}$ and any $\lambda>\lambda_{01}$, where $g=\left.v\right|_{\partial \Omega}$. 
In particular, we have the following for $r(t)$ and $g(t)=\left.r(t)\right|_{\partial \Omega}$ :

$$
\begin{aligned}
\int_{\Omega} e^{2 \tau \beta}|\nabla r(t)|^{2} d x \leq C & \left\{\tau \int_{\Omega} e^{2 \tau \beta} \beta\left|\left(1+x_{2}\right) k \wedge z(t)\right|^{2} d x\right. \\
& +\tau^{1 / 2} e^{2 \tau}\|g(t)\|_{1 / 2, \partial \Omega}^{2}+\tau^{2} \lambda^{2} \int_{B_{00}} e^{2 \tau \beta} \beta^{2}|r(t)|^{2} d x \\
& \left.+\int_{B_{00}} e^{2 \tau \beta}|\nabla r(t)|^{2} d x\right\} .
\end{aligned}
$$

To estimate the last integral in (3.38), let us introduce an open set $B_{01}$ such that $B_{00} \subset \subset B_{01} \subset \subset B_{0}$ and a function $\xi_{01} \in \mathcal{C}_{0}^{2}\left(B_{01}\right)$ such that

$$
0 \leq \xi_{01} \leq 1 \text { and } \xi_{01}=1 \text { in } B_{00} .
$$

Integrating by parts, it follows from (3.35) that

$$
\begin{aligned}
\int_{B_{00}} e^{2 \tau \beta}|\nabla r(t)|^{2} d x \leq & \int_{B_{01}} e^{2 \tau \beta} \xi_{01}|\nabla r(t)|^{2} d x \\
= & -\int_{B_{01}} e^{2 \tau \beta} \xi_{01} \operatorname{div}\left(\left(1+x_{2}\right) \mathrm{k} \wedge z\right)(t) r(t) d x \\
& -\frac{1}{2} \int_{B_{01}} e^{2 \tau \beta} \nabla \xi_{01} \cdot \nabla|r(t)|^{2} d x-\int_{B_{01}} \xi_{01} \nabla e^{2 \tau \beta} \cdot \nabla|r(t)|^{2} d x .
\end{aligned}
$$

Integrating again by parts, applying Young's inequality, and taking into account that $\left|\Delta\left(e^{2 \tau \beta} \xi_{01}\right)\right| \leq C \tau^{2} \lambda^{2} \beta^{2} e^{2 \tau \beta}$ for some positive constant $C$, after some straightforward computations we deduce that

$$
\int_{B_{00}} e^{2 \tau \beta}|\nabla r(t)|^{2} d x \leq C\left\{\tau^{2} \lambda^{2} \int_{B_{01}} e^{2 \tau \beta} \beta^{2}|r(t)|^{2} d x+\int_{B_{01}} e^{2 \tau \beta}|z(t)|^{2} d x\right\} .
$$

Replacing this inequality in (3.38), we obtain the following for each $t \in(0, T)$ :

$$
\begin{aligned}
\int_{\Omega} e^{2 \tau \beta}|\nabla r(t)|^{2} d x \leq C & \left\{\tau \int_{\Omega} e^{2 \tau \beta} \beta|z(t)|^{2} d x\right. \\
& +\tau^{1 / 2} e^{2 \tau}\|g(t)\|_{1 / 2, \partial \Omega}^{2} \\
& \left.+\tau^{2} \lambda^{2} \int_{B_{01}} e^{2 \tau \beta} \beta^{2}|r(t)|^{2} d x\right\} .
\end{aligned}
$$

Now, let us put $\tau=s /\left(t^{4}(T-t)^{4}\right)$ and let us choose $s>s_{02}=\max \left(s_{01}, \tau_{2}(T / 2)^{8}\right)$. Then $\tau>\tau_{2}$. Let us multiply by $\exp \left(-2 s \exp \left(2 \lambda\left\|\eta_{0}\right\|_{\infty}\right) /\left(t^{4}(T-t)^{4}\right)\right)$ the previous inequality and let us integrate with respect to $t$ in $(0, T)$. This leads to the estimate

$$
\begin{aligned}
\int_{0}^{T} \int_{\Omega} e^{-2 s \alpha}|\nabla r|^{2} d x d t \leq C & \left\{\int_{0}^{T} \int_{\Omega} e^{-2 s \alpha} s \varphi|z|^{2} d x d t\right. \\
& +\int_{0}^{T} e^{-2 s \alpha^{*}}\left(s \varphi^{*}\right)^{1 / 2}\|g(t)\|_{1 / 2, \partial \Omega}^{2} d t \\
& \left.+\int_{0}^{T} \int_{\omega_{1}} e^{-2 s \alpha}(s \lambda \varphi)^{2}|r|^{2} d x d t\right\}
\end{aligned}
$$

where $\alpha^{*}$ and $\varphi^{*}$ were introduced in section 3.1. 
The first term in the right-hand side of (3.39) can be absorbed by the left-hand side $I(s, \lambda ; z)$ in $(3.33)$ for $s$ large enough. Hence, we obtain

$$
\begin{aligned}
I(s, \lambda ; z) \leq C & \left\{\int_{0}^{T} \int_{\omega_{0}} e^{-2 s \alpha} s^{3} \lambda^{4} \varphi^{3}|z|^{2} d x d t+\int_{0}^{T} e^{-2 s \alpha^{*}}\left(s \varphi^{*}\right)^{1 / 2}\|g(t)\|_{1 / 2, \partial \Omega}^{2} d t\right. \\
& \left.+\int_{0}^{T} \int_{\omega_{1}} e^{-2 s \alpha}(s \lambda \varphi)^{2}|r|^{2} d x d t+\int_{0}^{T} \int_{\mathcal{O}} e^{-2 s \alpha}|\phi|^{2} d x d t\right\}
\end{aligned}
$$

for any $\lambda>\lambda_{01}$ and any $s>s_{03}$.

Step 3. Step 3 estimates the norm of the trace of the pressure on the boundary. To this end, we introduce three new functions:

$$
\chi(t)=e^{-s \alpha^{*}(t)}\left(s \varphi^{*}(t)\right)^{1 / 4}, \quad \tilde{z}=\chi(t) z, \quad \tilde{r}=\chi(t) r .
$$

From (3.32), we see that $(\tilde{z}, \tilde{r})$ satisfies

$$
\left\{\begin{array}{l}
-\tilde{z}_{t}-\Delta \tilde{z}+\tilde{z}+\nabla \tilde{r}=-\chi^{\prime} z+\chi\left(1+x_{2}\right) \mathrm{k} \wedge z+\chi \phi 1_{\mathcal{O}} \quad \text { in } Q \\
\operatorname{div} \tilde{z}=0 \text { in } Q \\
\tilde{z}=0 \text { on } \Sigma \\
\tilde{z}(T)=0 \text { in } \Omega
\end{array}\right.
$$

Using the continuity of the trace operator and standard a priori estimates for the pressure, we deduce that

$$
\begin{aligned}
\int_{0}^{T}\|\tilde{r}(t)\|_{1 / 2, \partial \Omega}^{2} d t \leq & \int_{0}^{T}\|\tilde{r}(t)\|_{1, \Omega}^{2} d t \\
\leq C & \left\{\int_{0}^{T} \int_{\Omega} e^{-2 s \alpha^{*}} s^{5 / 2}\left(\varphi^{*}\right)^{3}|z|^{2} d x d t\right. \\
& \left.+\int_{0}^{T} \int_{\mathcal{O}} e^{-2 s \alpha^{*}}\left(s \varphi^{*}\right)^{1 / 2}|\phi|^{2} d x d t\right\} .
\end{aligned}
$$

We have used here that $\left|\chi^{\prime}(t)\right|^{2} \leq C e^{-2 s \alpha^{*}} s^{5 / 2}\left(\varphi^{*}(t)\right)^{3}$ for all $t \in(0, T)$. We thus obtain a new estimate from (3.40):

$$
\begin{aligned}
I(s, \lambda ; z) \leq C & \left\{\int_{0}^{T} \int_{B_{00}} e^{-2 s \alpha} s^{3} \lambda^{4} \varphi^{3}|z|^{2} d x d t+\int_{0}^{T} \int_{B_{01}} e^{-2 s \alpha}(s \lambda \varphi)^{2}|r|^{2} d x d t\right. \\
& \left.+\int_{0}^{T} \int_{\mathcal{O}} e^{-2 s \alpha}(s \varphi)^{1 / 2}|\phi|^{2} d x d t\right\}
\end{aligned}
$$

for any $\lambda>\lambda_{01}$ and any $s>s_{04}$.

Step 4. It remains to estimate the local term in the right-hand side of (3.41) containing $|r|^{2}$ in terms of $z$ and $\phi$.

Assume that the pressure $r$ has been normalized in such a way that

$$
\int_{B_{01}} r(t) d x=0 \quad \forall t \in(0, T) .
$$

Then there exists $C>0$ such that

$$
\int_{B_{01}}|r(t)|^{2} d x \leq C \int_{B_{01}}|\nabla r(t)|^{2} d x \quad \forall t \in(0, T)
$$


and also

$$
\int_{0}^{T} \int_{B_{01}} e^{-2 s \alpha}(s \lambda \varphi)^{2}|r|^{2} d x d t \leq C \int_{0}^{T} \int_{B_{01}} e^{-2 s \widehat{\alpha}}(s \lambda \widehat{\varphi})^{2}|\nabla r|^{2} d x d t
$$

where the functions $\widehat{\alpha}=\widehat{\alpha}(t)$ and $\widehat{\varphi}=\widehat{\varphi}(t)$ were introduced in section 3.1.

From (3.32), we see that

$$
\begin{aligned}
\int_{0}^{T} \int_{B_{01}} e^{-2 s \widehat{\alpha}}(s \lambda \widehat{\varphi})^{2}|\nabla r|^{2} d x d t \leq C & \left\{\int_{0}^{T} \int_{B_{01}} e^{-2 s \widehat{\alpha}}(s \lambda \widehat{\varphi})^{2}\left(|z|^{2}+|\phi|^{2}\right) d x d t\right. \\
& \left.+\int_{0}^{T} \int_{B_{01}} e^{-2 s \widehat{\alpha}}(s \lambda \widehat{\varphi})^{2}\left(\left|z_{t}\right|^{2}+|\Delta z|^{2}\right) d x d t\right\} .
\end{aligned}
$$

Therefore, in view of (3.41), we obtain

$$
\begin{aligned}
I(s, \lambda ; z) \leq C & \left\{\int_{0}^{T} \int_{B_{01}} e^{-2 s \widehat{\alpha}}\left(s^{3} \lambda^{4} \widehat{\varphi}^{3}|z|^{2}+(s \lambda \widehat{\varphi})^{2}|\phi|^{2}\right) d x d t\right. \\
& +\int_{0}^{T} \int_{B_{01}} e^{-2 s \widehat{\alpha}}(s \lambda \widehat{\varphi})^{2}\left(\left|z_{t}\right|^{2}+|\Delta z|^{2}\right) d x d t \\
& \left.+\int_{0}^{T} \int_{\mathcal{O}} e^{-2 s \alpha}(s \varphi)^{1 / 2}|\phi|^{2} d x d t\right\} .
\end{aligned}
$$

Step 5. The rest of the proof deals with the estimates of the local integrals containing $|\Delta z|^{2}$ and $\left|z_{t}\right|^{2}$. First, we will be concerned with $|\Delta z|^{2}$.

Let us introduce a function $\xi_{0} \in \mathcal{C}_{0}^{4}\left(B_{0}\right)$ such that

$$
0 \leq \xi_{0} \leq 1 \text { and } \xi_{0}=1 \text { in } B_{01} .
$$

Let us set $\widehat{z}(x, t)=e^{-s \widehat{\alpha}} \widehat{\varphi} \xi_{0} \Delta z(T-t)$. We want to estimate the norm $\|\widehat{z}\|_{L^{2}\left(B_{01} \times(0, T)\right)^{2}}$. Following the arguments in [8] (see Step 4), we can deduce that

$$
\begin{aligned}
& \int_{0}^{T} \int_{B_{01}} e^{-2 s \widehat{\alpha}}(s \lambda \widehat{\varphi})^{2}|\Delta z|^{2} d x d t=\int_{0}^{T} \int_{B_{01}} s^{2} \lambda^{2}|\widehat{z}|^{2} d x d t \\
& \quad \leq C\left(\int_{0}^{T} \int_{B_{0}} e^{-2 s \widehat{\alpha}} s^{4} \lambda^{2} \widehat{\varphi}^{9 / 2}|z|^{2} d x d t+\int_{0}^{T} \int_{B_{0}} e^{-2 s \widehat{\alpha}}(s \lambda \widehat{\varphi})^{2}|\phi|^{2} d x d t\right) .
\end{aligned}
$$

Thus, from (3.42) we have

$$
\begin{aligned}
& I(s, \lambda ; z) \\
& \quad \leq C\left(\int_{0}^{T} \int_{B_{0}} e^{-2 s \widehat{\alpha}} s^{4} \lambda^{4} \widehat{\varphi}^{9 / 2}|z|^{2} d x d t+\int_{0}^{T} \int_{B_{0}} e^{-2 s \widehat{\alpha}}(s \lambda \widehat{\varphi})^{2}|\phi|^{2} d x d t\right. \\
& \left.\quad+\int_{0}^{T} \int_{B_{01}} e^{-2 s \widehat{\alpha}}(s \lambda \widehat{\varphi})^{2}|z|^{2} d x d t+\int_{0}^{T} \int_{\mathcal{O}} e^{-2 s \alpha}(s \varphi)^{1 / 2}|\phi|^{2} d x d t\right) .
\end{aligned}
$$

Step 6. Now we want to estimate $\left|z_{t}\right|^{2}$. Due to the regularity properties of $\phi$, we can use here a more straightforward argument than in [8], where the right-hand side belongs only to $L^{2}(Q)^{2}$. 
First, notice that

$$
\begin{aligned}
& \int_{0}^{T} \int_{B_{01}} e^{-2 s \widehat{\alpha}}(s \lambda \widehat{\varphi})^{2}\left|z_{t}\right|^{2} d x d t \leq \delta \int_{0}^{T} \int_{B_{01}} e^{-2 s \alpha} \frac{1}{s \varphi}\left|z_{t}\right|^{2} d x d t \\
& \quad+\delta \int_{0}^{T} \int_{B_{01}} e^{-2 s \alpha^{*}} \frac{1}{s^{3}\left(\varphi^{*}\right)^{7 / 2}}\left|z_{t t}\right|^{2} d x d t \\
& \quad+C_{\delta} \int_{0}^{T} \int_{B_{01}} e^{-4 s \alpha^{*}+2 s \alpha^{*}} s^{7} \lambda^{4} \widehat{\varphi}^{15 / 2}|z|^{2} d x d t .
\end{aligned}
$$

This is easily obtained by integrating by parts in time. We will later choose $\delta>0$ small enough.

We have the following auxiliary result.

Lemma 3.6. Let $(z, r)$ be the solution of (3.32). Then the following estimate holds:

$$
\begin{aligned}
& \int_{0}^{T} \int_{\Omega} e^{-2 s \alpha^{*}} \frac{1}{s^{3}\left(\varphi^{*}\right)^{7 / 2}}\left|z_{t t}\right|^{2} d x d t \\
& \quad \leq C\left(I(s, \lambda ; z)+\int_{0}^{T} \int_{\mathcal{O}} e^{-2 s \alpha^{*}}\left(\frac{1}{s \varphi^{*}}|\phi|^{2}+\frac{1}{s^{3}\left(\varphi^{*}\right)^{7 / 2}}\left|\phi_{t}\right|^{2}\right) d x d t\right) .
\end{aligned}
$$

Proof. Multiply (3.32) by $e^{-2 s \alpha^{*}} s^{-2}\left(\varphi^{*}\right)^{-9 / 4} z_{t t}$ and integrate in $Q$. Noticing that

$$
\left|\left(e^{-2 s \alpha^{*}}\left(\varphi^{*}\right)^{-9 / 4}\right)_{t}\right| \leq C e^{-2 s \alpha^{*}} s\left(\varphi^{*}\right)^{-1},
$$

after some computations we deduce that

$$
\begin{aligned}
& \int_{0}^{T} \int_{\Omega} e^{-2 s \alpha^{*}} \frac{1}{s^{2}\left(\varphi^{*}\right)^{9 / 4}}\left|\nabla z_{t}\right|^{2} d x d t \\
& \leq C\left\{\int_{0}^{T} \int_{\Omega} e^{-2 s \alpha^{*}} \frac{1}{s \varphi^{*}}\left(|z|^{2}+\left|z_{t}\right|^{2}\right) d x d t\right. \\
& \left.\quad+\int_{0}^{T} \int_{\Omega} e^{-2 s \alpha^{*}} s\left(\varphi^{*}\right)^{1 / 4}|\nabla z|^{2} d x d t+\int_{0}^{T} \int_{\mathcal{O}} e^{-2 s \alpha^{*}} \frac{1}{s \varphi^{*}}|\phi|^{2} d x d t\right\} \\
& \quad+\frac{1}{2} \int_{0}^{T} \int_{\Omega} e^{-2 s \alpha^{*}} \frac{1}{s^{3}\left(\varphi^{*}\right)^{7 / 2}}\left|z_{t t}\right|^{2} d x d t .
\end{aligned}
$$

On the other hand, if we compute the time derivative of (3.32) and then we multiply the result by $e^{-2 s \alpha^{*}} s^{-3}\left(\varphi^{*}\right)^{-7 / 2} z_{t t}$, we find that

$$
\begin{aligned}
& \int_{0}^{T} \int_{\Omega} e^{-2 s \alpha^{*}} \frac{1}{s^{3}\left(\varphi^{*}\right)^{7 / 2}}\left|z_{t t}\right|^{2} d x d t \\
& \quad \leq \int_{0}^{T} \int_{\Omega} e^{-2 s \alpha^{*}} \frac{1}{s^{2}\left(\varphi^{*}\right)^{9 / 4}}\left|\nabla z_{t}\right|^{2} d x d t \\
& \quad+C\left(\int_{0}^{T} \int_{\Omega} e^{-2 s \alpha^{*}} \frac{1}{s \varphi^{*}}\left|z_{t}\right|^{2} d x d t+\int_{0}^{T} \int_{\mathcal{O}} e^{-2 s \alpha^{*}} \frac{1}{s^{3}\left(\varphi^{*}\right)^{7 / 2}}\left|\phi_{t}\right|^{2} d x d t\right)
\end{aligned}
$$

From (3.46) and (3.47), we see that (3.45) holds. 
In view of this lemma, we have

$$
\begin{aligned}
& \int_{0}^{T} \int_{B_{01}} e^{-2 s \widehat{\alpha}}(s \lambda \widehat{\varphi})^{2}\left|z_{t}\right|^{2} d x d t \\
& \quad \leq C \delta\left(I(s, \lambda ; z)+\int_{0}^{T} \int_{\mathcal{O}} e^{-2 s \alpha^{*}}\left(\frac{1}{s \varphi^{*}}|\phi|^{2}+\frac{1}{s^{3}\left(\varphi^{*}\right)^{7 / 2}}\left|\phi_{t}\right|^{2}\right) d x d t\right) \\
& \quad+C_{\delta} \int_{0}^{T} \int_{B_{01}} e^{-4 s \alpha^{*}+2 s \alpha^{*}} s^{7} \lambda^{4} \widehat{\varphi}^{15 / 2}|z|^{2} d x d t .
\end{aligned}
$$

If we assume that $\gamma_{1}<1$, then $\left(3-\gamma_{1}\right) / 2>1$, and from Lemma 3.2 we deduce that $\left(3-\gamma_{1}\right) \widehat{\alpha} / 2>\alpha^{*}$ for sufficiently large $\lambda$, say, $\lambda>\lambda_{02}$. Consequently, $-4 \widehat{\alpha}+2 \alpha^{*}<$ $-\left(1+\gamma_{1}\right) \widehat{\alpha}$ and

$$
\begin{aligned}
& \int_{0}^{T} \int_{B_{01}} e^{-2 s \widehat{\alpha}}(s \lambda \widehat{\varphi})^{2}\left|z_{t}\right|^{2} d x d t \\
& \quad \leq C \delta\left(I(s, \lambda ; z)+\int_{0}^{T} \int_{\mathcal{O}} e^{-2 s \alpha^{*}}\left(\frac{1}{s \varphi^{*}}|\phi|^{2}+\frac{1}{s^{3}\left(\varphi^{*}\right)^{7 / 2}}\left|\phi_{t}\right|^{2}\right) d x d t\right) \\
& \quad+C_{\delta} \int_{0}^{T} \int_{B_{01}} e^{-\left(1+\gamma_{1}\right) s \widehat{\alpha}} s^{7} \lambda^{4} \widehat{\varphi}^{15 / 2}|z|^{2} d x d t
\end{aligned}
$$

for any $\lambda>\lambda_{02}$ and any $s>s_{04}$.

From (3.44) and this estimate, choosing $\delta>0$ small enough, we find

$$
\begin{aligned}
& I(s, \lambda ; z) \\
& \leq C\left\{\int_{0}^{T} \int_{B_{0}} e^{-\left(1+\gamma_{1}\right) s \widehat{\alpha}} s^{7} \lambda^{4} \widehat{\varphi}^{15 / 2}|z|^{2} d x d t+\int_{0}^{T} \int_{B_{0}} e^{-2 s \widehat{\alpha}}(s \lambda \widehat{\varphi})^{2}|\phi|^{2} d x d t\right. \\
& \left.\quad+\int_{0}^{T} \int_{\mathcal{O}} e^{-2 s \alpha}\left((s \varphi)^{1 / 2}|\phi|^{2}+\frac{1}{s^{3}\left(\varphi^{*}\right)^{7 / 2}}\left|\phi_{t}\right|^{2}\right) d x d t\right\}
\end{aligned}
$$

for all $\lambda>\lambda_{02}$ and $s>s_{04}$.

Obviously, this yields (3.8). The proof of (3.9) is very similar and in fact much simpler, since the left-hand side of (2.1) is zero.

Thus, we have proved Lemma 3.4 for $\lambda_{1}=\lambda_{02}$ and $s_{1}=s_{04}$ (two parameters depending on $\Omega, \omega, \mathcal{O}$, and $T)$.

4. Some final remarks. The geometrical hypothesis $\omega \cap \mathcal{O} \neq \emptyset$ is required to prove the existence of both $\varepsilon$-insensitizing and insensitizing controls. In the first case, this assumption is used to prove a unique continuation property (Lemma 2.1). In the case of insensitizing controls, it is used to prove an observability inequality. The problem is completely open when $\omega \cap \mathcal{O}=\emptyset$ (see [18]).

The existence of insensitizing controls is guaranteed by the null controllability property of a cascade system of quasi-geostrophic equations (1.5)-(1.6). In this case, the control acts indirectly on one variable through the other one. Of course, this controllability property is stronger than the null controllability of a single quasigeostrophic system. 
To prove the null controllability property of the cascade system, there are two main difficulties.

First is the need for a unique continuation result for the adjoint system (2.2)-(2.1). The presence of the Coriolis term permits us to relate the second component of the velocity and its associated vorticity, and this is a key point in the proof of uniqueness (see Remark 2).

The second problem is the need for an observability inequality for the adjoint. This inequality comes from an appropriate (global) Carleman estimate. The main idea is to estimate $\phi$ in terms of $\operatorname{curl} \phi$ in a ball contained in $\omega \cap \mathcal{O}$ in the right-hand side of (3.10). This is possible again due to the presence of the Coriolis term (in fact, our method does not work in the case of the usual Stokes equations). We rewrite the system using the stream function and the vorticity and we see that the Coriolis term leads to an expression of the horizontal derivative of the stream function in terms of the vorticity. In this way, we are able to avoid estimates of pressure terms, which are in general very hard to deduce (see the appendix in section 3). Moreover, the weight in the right-hand side of (3.10) is larger than the weight in the left-hand side. Accordingly, the terms in the right cannot be absorbed directly as in the case of the heat equation (see [18]) and this fact requires some additional work.

Acknowledgments. The authors wish to thank J.-P. Puel of the Laboratoire de Mathématiques Appliquées, Université Versailles/Saint Quentin-en-Yvelines (France) and S. Guerrero of the Departamento de Ecuaciones Diferenciales y Análisis Numérico, Universidad de Sevilla (Spain), for very useful discussions.

\section{REFERENCES}

[1] R. Bermejo And P. G. Del Sastre, Numerical studies of the long-term dynamics of the $2 D$ Navier-Stokes equations applied to ocean circulation, in XVII CEDYA: Congress on Differential Equations and Applications, Universidad de Salamanca, 2001, pp. 15-34.

[2] O. Bodart AND C. FABRE, Controls insensitizing the norm of the solution of a semilinear heat equation, J. Math. Anal. Appl., 195 (1995), pp. 658-683.

[3] O. Bodart, M. González-Burgos, and R. PÉrez-García, Insensitizing controls for a semilinear heat equation with a superlinear nonlinearity, C. R. Math. Acad. Sci. Paris, 335 (2002), pp. 677-682.

[4] C. FABRE, Uniqueness results for Stokes equations and their consequences in linear and nonlinear control problems, ESAIM Control Optim. Calc. Var., 1 (1996), pp. 267-302.

[5] C. Fabre and G. Lebeau, Régularité et unicité pour le problème de Stokes, Comm. Partial Differential Equations, 27 (2002), pp. 437-475.

[6] C. Fabre, J.-P. Puel, and E. Zuazua, Approximate controllability of the semilinear heat equation, Proc. Roy. Soc. Edinburgh Sect. A, 125 (1995), pp. 31-61.

[7] E. Fernández-Cara, G. C. García, And A. Osses, Insensitizing controls for a large-scale ocean circulation model, C. R. Math. Acad. Sci. Paris, 337 (2003), pp. 265-270.

[8] E. Fernández-Cara, S. Guerrero, O. Y. Imanuvilov, and J.-P. Puel, Local exact controllability of the Navier-Stokes system, J. Math. Pures Appl. (9), 83 (2004), pp. 1501-1542.

[9] A. Fursikov and O. Y. Imanuvilov, Controllability of Evolution Equations, Lecture Notes Ser. 34, Research Institute of Mathematics, Seoul National University, Korea, 1996.

[10] O. Y. ImANuvilov, On exact controllability of Navier-Stokes equations, ESAIM Control Optim. Calc. Var., 3 (1998), pp. 97-131.

[11] O. Y. Imanuvilov, Remarks on exact controllability for the Navier-Stokes equations, ESAIM Control Optim. Calc. Var., 6 (2001), pp. 39-72.

[12] O. Y. Imanuvilov and J.-P. Puel, Global Carleman estimates for weak solutions of elliptic non homogeneous Dirichlet problems, Int. Math. Res. Not., 16 (2003), pp. 883-913.

[13] J. L. Lions, Quelques notions dans l'analyse et le contrôle de systèmes à données incomplètes, in Proceedings of the XIth Congress on Differential Equations and Applications/First Congress on Applied Mathematics, University of Málaga, 1990, pp. 43-54.

[14] J. L. Lions, Remarks on approximate controllability, J. Anal. Math., 59 (1992), pp. 103-116. 
[15] G. I. Marchuk, V. I. Agoshkov, and V. P. Shutyaev, Adjoint Equations and Perturbation Algorithms in Nonlinear Problems, CRC Press, Boca Raton, FL, 1996.

[16] P. G. Myers and A. J. Weaver, A diagnostic barotropic finite-element ocean circulation model, J. Atmos. Ocean Tech., 12 (1995), pp. 511-526.

[17] R. Temam, Navier-Stokes Equations, 2nd ed., North-Holland, Amsterdam, 1984.

[18] L. DE TERESA, Insensitizing control for a semilinear heat equation, Comm. Partial Differential Equations, 25 (2000), pp. 39-72. 